\title{
Effektive Blutzuckerregulation über die Niere
}

Die Optimierung der Blutzuckerkontrolle unter Beachtung von Begleiterkrankungen und Risikofaktoren ist das Ziel der individualisierten Diabetestherapie. Potenzielle Nebenwirkungen einer pharmakologischen antiglykämischen Therapie, wie erhöhtes Hypoglykämierisiko und Gewichtszunahme, limitieren oftmals den Erfolg. Die Hemmung der renalen Glukosereabsorption mit Empagliflozin ist eine effektive Therapieoption, um den Blutzucker langanhaltend und hypoglykämiearm zu senken.

Das Therapieprinzip beruht auf der Inhibierung des Natrium-Glucose-Cotransporters (SGLT2) in der Niere, einem Transportprotein, das für ca. 90\% der Reabsorption der glomerulär filtrierten Glukose aus dem proximalen Tubulus in den Blutkreislauf verantwortlich ist. Bei chronisch gesteigerten Glukosespiegeln ist SGLT2 vermehrt aktiv, was die Nierenschwelle für Glukose ansteigen lässt und zum erhöhten Blutzuckerspiegel beiträgt. Durch die selektive Hemmung von SGLT2 wird die Nierenschwelle für die Glukosereabsorption abgesenkt und damit täglich vermehrt Glukose über den Urin ausgeschwemmt. Diese therapeutisch induzierte Glukosurie verbessert die Blutzuckerkontrolle, kombiniert mit einer moderaten Gewichts- und Blutdrucksenkung**. Da dieser Mechanismus nicht vom Insulin abhängt, besteht die
Möglichkeit eines breiten Einsatzes in jedem Krankheitsstadium des Typ-2Diabetes und unabhängig von der Betazellfunktion [1-3].

Im Mai 2014 wurde der SGLT2-Hemmer Empagliflozin europaweit zur Typ-2-Diabetestherapie zugelassen. In Deutschland kann Empagliflozin unter dem Namen Jardiance ${ }^{\circledR}$ seit August 2014 in den Dosierungen $10 \mathrm{mg}$ und $25 \mathrm{mg}$ verordnet werden. Empagliflozin ist als Monotherapie angezeigt, wenn durch Diät und Bewegung keine ausreichende glykämische Kontrolle erreicht werden kann und eine Metformin-Unverträglichkeit vorliegt. Zudem kann es unter bestimmten Voraussetzungen mit anderen Antidiabetika einschließlich Insulin kombiniert werden [4].

Überzeugende Daten in verschiedenen Therapieregimen

Auf der Einführungspressekonferenz wurden die Ergebnisse eines beeindruckenden Studienprogramms mit mehr als 13000 Patienten präsentiert $[5,6]$.

In der Monotherapie erwies sich Empagliflozin als wirksamer SGLT2-Hemmer. So sank der $\mathrm{HbA}_{1 \mathrm{c}}$-Wert nach einer 24-wöchigen Behandlung mit Empagliflozin $10 \mathrm{mg}$ und $25 \mathrm{mg}$ gegenüber Placebo statistisch signifikant um 0,74 respektive $0,85 \%$ und damit in einer vergleichbaren Größenordnung wie mit dem DPP-4-Hemmer Sitagliptin $100 \mathrm{mg}$
[7]. Bei Patienten mit deutlich unzureichend eingestelltem Blutzucker fiel die Wirkung von Empagliflozin effektiver aus. So kam es bei Patienten mit einem $\mathrm{HbA}_{1 \mathrm{c}}$-Ausgangswert $\geq 8,5 \%$ mit beiden Dosierungen zur Absenkung des $\mathrm{HbA}_{1 \mathrm{c}}{ }^{-}$ Wertes um 1,4\% [7]. Dieser Effekt war auch einer Behandlung mit $100 \mathrm{mg}$ Sitagliptin signifikant überlegen. Lag der $\mathrm{HbA}_{1 \mathrm{c}}$-Ausgangswert bei über $10 \% \mathrm{kam}$ es mit Empagliflozin sogar $\mathrm{zu}$ einer $\mathrm{HbA}_{1 \mathrm{c}}$ Senkung von bis zu 3,7\% [7].

Im direkten Vergleich mit dem Sulfonylharnstoff Glimepirid (durchschnittliche Dosis nach 2 Jahren: 2,7 mg) bewies Empagliflozin ( $25 \mathrm{mg}$ ) eine anhaltende Wirkung auf die Blutzuckerkontrolle. In der zweijährigen Studie mit 1545 Erwachsenen mit Typ-2-Diabetes wurden beide Medikamente jeweils als Add-on zu Metformin gegeben. Nach zweijähriger Behandlungsdauer fiel die $\mathrm{HbA}_{1 \mathrm{c}}{ }^{-}$ Senkung von Empagliflozin mit -0,11\% signifikant deutlicher aus als die von Glimepirid [4].

Frühzeitige Intensivierung der Blutzuckertherapie

Verschiedene Interventionsstudien haben den Effekt einer frühzeitigen intensivierten Blutzuckertherapie auf die kardiovaskuläre Risikoreduktion belegt [8]. Offensichtlich führt die frühe intensive Therapie zu einer Beeinflussung des me-

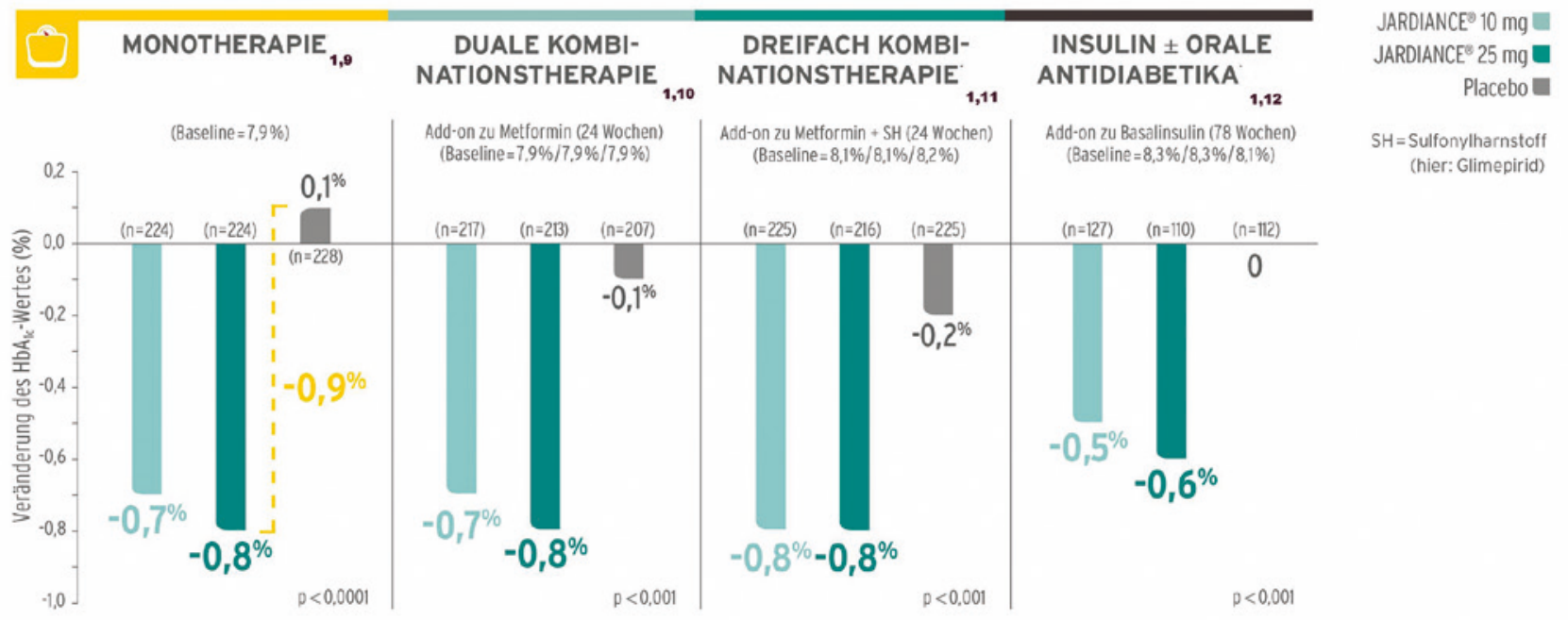

Monotherapie: $\mathrm{HbA}_{1 \mathrm{c}}$-Senkung von $1,4 \%$ bei $\mathrm{HbA}_{1 \mathrm{c}}$-Ausgangswerten $\geq 8,5 \%$ 1,

Abb. 1 Senkung des $\mathrm{HbA}_{1 \mathrm{c}}$-Wertes in verschiedenen Therapieregimen. 
Empagliflozin im Schnellcheck

- Jardiance ${ }^{\circledR}$ ist zur Behandlung von Typ-2-Diabetes bei Erwachsenen sowohl als Monotherapie bei Metformin-Unverträglichkeit als auch als Add-on-Kombinationstherapie mit anderen blutzuckersenkenden Arzneimitteln einschließlich Insulin zugelassen

- Empagliflozin führt in der Monotherapie und in Kombination mit verschiedenen oralen Therapien und Insulin zu signifikanten $\mathrm{HbA}_{1 c}$ Absenkungen.

- Die Filmtabletten müssen nur einmal täglich unabhängig von den Mahlzeiten eingenommen werden.

- Die empfohlene Anfangsdosis für Empagliflozin beträgt $10 \mathrm{mg}$ einmal täglich.

- Bei Patienten, die Empagliflozin $10 \mathrm{mg}$ einmal täglich vertragen, eine eGFR $\geq 60 \mathrm{ml}$ $\mathrm{min} / 1,73 \mathrm{~m}^{2}$ haben und eine engere Blutzuckerkontrolle benötigen, kann die Dosis auf $25 \mathrm{mg}$ einmal täglich erhöht werden.

tabolischen Gedächtnisses. Die Empagliflozinkombination mit Metformin oder mit Metformin und Sulfonylharnstoff als orale Zweifach-, respektive Dreifachtherapie, reduzierte den $\mathrm{HbA}_{1 \mathrm{c}}$ stärker als die jeweilige Ausgangsbehandlung [9, 10]. Nach Matthaei bietet Empagliflozin daher die Möglichkeit einer zeitnahen und effektiven Therapieeskalation mit Nichtinsulinen.

Die zusätzliche Gabe von Empagliflozin bei unzureichender glykämischer Kontrolle unter Insulin und Metformin ist eine weitere interessante Option, so Kaiser. In einer 78-wöchigen Studie mit insgesamt 453 adipösen Erwachsenen mit Typ-2-Diabetes und unzureichend eingestellten Blutzuckerspiegeln, mit einem durchschnittlichen $\mathrm{HbA}_{1 \mathrm{c}}$-Ausgangswert von $8,3 \%$, führte die Gabe von Empagliflozin $10 \mathrm{mg}$ bzw. $25 \mathrm{mg}$ als Add-on zu Basalinsulin $\mathrm{zu}$ einer signifikante $\mathrm{HbA}_{1 \mathrm{c}}{ }^{-}$ Senkung in beiden Empagliflozingruppen. Zudem konnte die tägliche Insulindosis verringert werden [11].

\section{Erwünschte Nebeneffekte}

auf Körpergewicht und Blutdruck*†

Bereits mit der ersten Empagliflozindosis wird die Glukoseausscheidung über die Niere angekurbelt und erreicht im Durchschnitt etwa 78g/Tag [4]. Durch die Glukosurie und dem damit verbundenen Energieverlust kommt es zu einer oft erwünschten und, unabhängig von der Begleitmedikation, signifikanten Gewichtsreduktion*. In der Sulfonylharnstoff-Vergleichsstudie sank in der Empagliflozingruppe das Körpergewicht im Mittel um 3,1 kg, während es in der Glimepiridgruppe um 1,3 kg zunahm [4]. Auch in der Add-on Behandlung zu Basalinsulin verringerte sich das Gewicht mit Empagliflozin im Durchschnitt um $2 \mathrm{~kg}$ und blieb auch nach 78 Wochen auf diesem niedrigeren Niveau. Im Vergleich dazu stieg in der Placebo-Gruppe das Körpergewicht um 0,4 kg an [11].
Eine moderate Reduktion des systolischen Blutdrucks ${ }^{\dagger}$ beruht wohl auf der mit der Glukosurie verbundenen milden Diurese, die unabhängig von der Begleitmedikation erfolgt [7, 9-12]. Das Ausmaß der Blutdrucksenkung unter Empagliflozin ist dabei abhängig vom Ausgangswert. Bei Patienten mit unkontrolliertem Blutdruck $(\geq 130 / 80 \mathrm{mmHg})$ fällt die Reduktion deutlich aus, während es bei Patienten mit kontrolliertem Blutdruck kaum zu Veränderungen kommt [13].

\section{Gute Verträglichkeit}

Empagliflozin hat ein günstiges Verträglichkeitsprofil mit einer Nebenwirkungsrate auf Placeboniveau [4]. Daten aus 4 gepoolten Phase-III-Studien zeigten, dass kein substanzeigenes Hypoglykämie-Risiko besteht [4, 9-11, 13]. Hypoglykämien traten in den Untersuchungen lediglich unter Kombinationstherapie mit Sulfonylharnstoff oder Insulin auf. Um das Risiko einer Hypoglykämie zu senken, kann daher in der Add-on Therapie mit Sulfonylharnstoff oder Insulin eine niedrigere Dosis der Sulfonylharnstoffe oder des Insulins erwogen werden [4].

Eine signifikante Glukosurie mit SGLT2Hemmern kann nur dann erzielt werden, wenn noch eine gute Nierenfunktion besteht. Mit abnehmender Nierenfunktion verringert sich die Menge der frei filtrierten Glukosemenge im Primärharn und entsprechend sinkt der blutzuckersenkende Effekt dieser Medikamente [14]. Daher sollte bei Patienten mit einer eGFR $<60 \mathrm{ml} / \mathrm{min} / 1,73 \mathrm{~m}^{2}$ keine Therapie mit Empagliflozin begonnen und bei einer persistierenden eGFR $<45 \mathrm{ml} / \mathrm{min} / 1,73 \mathrm{~m}^{2}$ aufgrund mangelnder Wirksamkeit abgesetzt werden [4].

Die Häufigkeit von Harnwegsinfektionen war insgesamt gering und lag bei mit 10 mg Empagliflozin behandelten Patienten nur sehr geringfügig über Placeboniveau. Die Intensität der überwiegend nur einmaligen Ereignisse war in den meisten Fällen leicht bis moderat [15]. Die Inzidenz von Infektionen des Genitaltrakts war bei den mit Empagliflozin behandelten Patienten höher als mit Placebo. Die Infektionen waren von leichter bis mittlerer Intensität und traten in den meisten Fällen nur einmalig, bei Frauen wesentlich häufiger als bei Männern auf [15]. Die beobachteten Harnwegsinfektionen und Infektionen des Genitaltrakts sind in den meisten Fällen mit lokalen oder systemischen Standardtherapien gut behandelbar [16].

Richard Kessing, Zeiskam

\footnotetext{
* Empagliflozin ist nicht zur Gewichtsabnahme zugelassen.

† Empagliflozin ist nicht zur Blutdrucksenkung zugelassen.
}

\section{Literatur}

1 Abdul-Ghani MA, DeFronzo RA. Endocrine Practice. 2008; 14: 782-790

2 DeFronzo RA. Diabetes Obes Metab 2012; 14: 5-14

3 Ferrannini E et al. Nat Rev Endocrinol 2012; 8: 495-502

4 JARDIANCE ${ }^{\circledR} 10 \mathrm{mg} /-25 \mathrm{mg}$ Fachinformation, Stand: Mai 2014

5 ClinicalTrials.gov. Phase III trials of empagliflozin/BI10773. http://clinicaltrials.gov. Stand: Juni 2014

6 Data on file

7 Roden $\mathrm{M}$ et al. Lancet Diabetes Endocrinol. 2013; 1: 208-219

8 Del Prato et al. TOSCA.IT Study Group. Lancet 2014; 384: 1096

9 Häring HU et al. Diabetes Care 2013; 36: 3396-3404

10 Häring HU et al. Diabetes Care 2014; published online ahead of print, doi:

11 Rosenstock J et al. Can J Diabetes 2013 Oct. 37 S4: S32

12 Kovacs C et al. Diabetes Obes Metab 2013; 16: $147-158$

13 Hach T et al. European Association for the Study of Diabetes (EASD), September 2327, Barcelona, Poster: 943

14 Barnett $A$ et al. Lancet Diabet Endocrinol 2014 doi: 10.1016/S2213-8587(13)70208-0

$15 \mathrm{Kim} \mathrm{G}$ et al. American Diabetes Association (ADA) $73^{\text {rd }}$ Scientific Sessions, 21-25 June 2013, Chicago, IL, USA (Poster 74-LB), 10.2337/dc13-2105.

16 Geerlings $S$ et al. Diabetes Res Clin Pract 2014; 3: 373-381

Quelle: Launch-Presseveranstaltung: „Empagliflozin - Reduktion der Glucoselast unabhängig von Insulin“. Ingelheim, 2. Juli 2014.

Der Text entstand mit freundlicher Unterstützung durch Boehringer Ingelheim $\mathrm{GmbH}$, Ingelheim und Lilly Deutschland $\mathrm{GmbH}$, Bad Homburg 